\title{
European Forest Types. Categories and types for sustainable forest management reporting and policy
}

\section{Luigi Portoghesi}

Abstract: Some considerations are reported on the Environmental European Agency, Technical report N. 9 by Barbati A, Corona P and Marchetti M: "Categories and types for sustainable forest management reporting and policy".

Keywords: Forest type, Management, Sustainability, European policy, Indicators.

Citation: Portoghesi L, 2006. European Forest Types. Categories and types for sustainable forest management reporting and policy. Forest@ 3 (4): 462-463. [online] URL: http://www.sisef.it/.

Environmental European Agency, Technical report No. 9 - Copenhagen, 2006. “European Forest types: Categories and types for sustainable forest management reporting and policy". Anna Barbati, Piermaria Corona, Marco Marchetti (ISBN 92-9167886-4; ISSN 1725-2237; [online] URL: http://reports.eea.europa.eu/technical_report_2006_9/en.

Recentemente pubblicato dall'Agenzia Europea per l'Ambiente (European Environment Agency EEA), il rapporto EUROPEAN FOREST TYPES - Categories and types for sustainable forest management reporting and policy presenta i risultati di uno studio condotto da un consorzio internazionale di esperti coordinato dall'Accademia Italiana di Scienze Forestali per la proposizione di una classificazione tipologica delle foreste Europee da adottare in sede MCPFE (Ministrial Conference on the Protection of Forest in Europe) nel reporting sulla Gestione Forestale Sostenibile (GFS) in Europa.

Il processo MCPFE comprende, infatti, tra le sue attività la pubblicazione di rapporti periodici sullo stato delle foreste e della gestione forestale nella regione pan-Europea, compilati a partire da dati su indicatori quantitativi e qualitativi di GFS forniti da National Focal Points dei 44 Paesi del continente Europeo. La formulazione di sette indicatori quantitativi inclusi nel sistema MCPFE (Forest area, Growing stock, Age structure and/or diameter distribution, Forest damage, Tree species composition, Naturalness, Deadwood) richiede infatti una restituzione per forest types. Il break-down dei dati nazionali su tali indicatori per forest types dovrebbe incrementare la specificità dell'informazione riportata e, dunque, l'informatività e la possibilità di interpretazione del dato stesso. Sinora, tuttavia, le classi di forest types utilizzate per il reporting sono talmente ampie e generiche (boschi di conifere, latifoglie e misti) da rendere pressoché priva di senso tale suddivisione; ciò soprattutto nei paesi, come l'Italia, caratterizzati da forte eterogeneità biogeografica, climatica, ecologica e dei sistemi selvicolturali e, di conseguenza, da notevole diversità fisionomico-strutturale delle formazioni forestali.

La tipologia forestale proposta (European Forest Types) nasce per superare tali limiti. L'obiettivo è concordare uno schema di classificazione delle foreste della regione pan-Europea (1004 milioni di ettari) che ne rifletta la varietà compositiva e strutturale, nonché il grado di naturalità così come definito dall'apposito indicatore MCPFE (foresta indisturbata, semi-naturale, piantagione forestale). Uno schema tipologico sufficientemente dettagliato per stratificare la diversità dei contesti ecologici e delle influenze antropiche che determinano e spiegano la variabilità degli indicatori in oggetto a scala continentale e, al contempo, facilmente implementabile a partire da dati inventariali o altrimenti raccolti nella pianificazione forestale.

Nel rapporto sono discussi i limiti di applicabilità di classificazioni già esistenti (classificazione degli habitat europei EUNIS, inquadramenti su base fitosociologica della vegetazione forestale europea) e vengono presentati i concetti e i criteri di delineazione adottati nello sviluppo degli European Forest Types.

La struttura della classificazione è gerarchica e articolata in due livelli: 14 classi (Categories) da utilizzare per il reporting MCPFE; 75 Types che corrispondono 
a un'ulteriore suddivisione della categoria in unità fisionomiche più di dettaglio, intese quali classi di raccordo per uno scaling-up dei dati nazionali alle 14 categorie di reporting.

Le categorie, segnatamente, corrispondono a classi di foreste considerabili relativamente omogenee al loro interno a scala continentale, per fattori climatici, edafici e grado di naturalità. Di queste, undici classi sono formazioni dominate da specie forestali native in Europa (Cat. 1-10) - la cui fisionomia è principalmente determinata dalla zonazione latitudinale e altitudinale della vegetazione forestale europea - e due classi sono rappresentate da formazioni azonali (Cat. 11-12). Nel complesso, queste tredici categorie sono largamente rappresentate da boschi semi-naturali.

Le piantagioni forestali (di specie native e non) e le formazioni naturalizzate di specie esotiche (i cosiddetti novel ecosystems) costituiscono una categoria separata (Cat. 14). In entrambe i casi si tratta di formazioni la cui struttura, composizione e funzionalità è originata e/o strettamente modellata dall'azione antropica.

Ogni categoria è caratterizzata da una specifica interazione tra fattori naturali (clima, suolo, regime idrologico) e antropici (sistemi selvicolturali o agrosilvo-pastorali), che riduce e riconduce il campo di variabilità degli indicatori MCPFE (in particolare: provvigione, distribuzione delle classi diametriche o delle età, composizione arborea, necromassa) entro un range caratteristico. La possibilità di riferire gli indicatori, attraverso le categorie, ai loro determinanti naturali e antropici aumenta le possibilità di valutazione e interpretazione dei valori stessi, soprattutto in una prospettiva di monitoraggio dei risultati della gestione forestale.

L'identificazione delle classi a livello operativo è guidata da una chiave dicotomica di classificazione, basata su informazioni ordinariamente disponibili nel contesto di un inventario forestale o di piani di gestione forestale (principalmente, la composizione arborea dei popolamenti forestali e i caratteri ecologici della stazione). La chiave dicotomica è integrata da una dettagliata chiave nomenclaturale che definisce le caratteristiche principali delle foreste afferenti a ciascuna categoria, la loro distribuzione geografica in Europa e i principali tipi in cui essa si manifesta. A livello di tipo è stabilito anche un collegamento con altri sistemi di classificazione degli habitat forestali EUNIS e Natura 2000.

Prima di essere pubblicato, lo schema di European Forest Types è stato sottoposto a un processo di peer revision da parte dell' EIONET Primary Contact Points (PCPs) and National Reference Centers (NRCs) for Nature Protection and Biodiversity.

L'accettazione della proposta da parte del processo MCPFE è stata avviata con successo attraverso la presentazione degli European Forest Types al Workshop MCPFE on pan-European understanding of forest classification tenutosi a Bled (Slovenia) dal 13 al 15 November 2006. In questa sede, National Focal Points dei paesi convenuti e rappresentanti dell'European Commission hanno concordato un documento finale in cui si raccomanda l'adozione formale delle categorie per il reporting MCPFE, da deliberare al prossimo MCPFE Expert Level Meeting previsto nel 2007.

\section{Author's box}

Luigi Portoghesi è professore associato di Dendrometria all'Università della Tuscia dove svolge ricerche sulla gestione multifunzionale delle foreste. E-mail: lporto@unitus.it 